\title{
Off-centred impact of droplets on horizontal cylinders with large curvature ratios
}

\author{
D. Jordan Bouchard ${ }^{* 1}$, Khalil Sidawi ${ }^{1}$, Sanjeev Chandra ${ }^{1}$ \\ ${ }^{1}$ Department of Mechanical and Industrial Engineering, University of Toronto, Toronto, \\ Canada \\ Corresponding author email: jordan.bouchard@mail.utoronto.ca
}

\begin{abstract}
Drop impact on cylindrical objects is a natural phenomenon with important industrial applications, e.g., mesh-style mist eliminators, where the goal is to capture drops on the mesh wires. The impact velocity of the drop on a cylinder is one factor that determines the amount of a drop that is captured, with drops impacting at greater than a threshold velocity only being partially captured by the fibre. The threshold velocity will change depending on how offcentered (eccentric) the drop impacts the cylinder, and the ratio of the fibre and drop diameters, known as the curvature ratio, $R^{*}$. Currently, little experimental data exists for the threshold velocity of drops impacts in the range of $\mathrm{We}<14$ and curvature ratios $R^{*}>1$, which this study is designed to provide. This parameter space is equivalent to drops on the order of 10 microns impacting fibers with diameters on the order of 100 microns. We show that the threshold velocity of capture decreases for increasing eccentricity and increases for increasing $R^{*}$. This is in agreement with results for droplet impacts where the $R^{*}<1$.
\end{abstract}

Keywords: fibre; fiber; threshold velocity, mesh, air-liquid separation

\section{Introduction}

Droplet impact on cylindrical objects occurs in nature, e.g., rain on plant stems, and is relevant to many industrial applications, e.g., filtration, mist elimination, liquid recovery. There are several experimental and numerical studies devoted to this topic, and droplet impact on horizontal cylindrical objects is well understood. Droplet impact on cylinders will produce three different regimes: droplet disintegration or splitting, momentum induced dripping, and gravity induced dripping [1]. Every cylinder has a maximum amount of liquid that it can capture before gravity induced dripping occurs. This volume increases with the diameter of the cylinder and can be predicted with reasonable accuracy from analytical models $[1,2]$. Of particular interest to the research community is the threshold impact velocity, $U_{c}$, which determines how much of the drop will be captured by cylinders. At impact velocities below the $U_{c}$ the entire drop will be captured; at velocities above the $U_{c}$, the volume captured by the cylinder decreases [2-4].

Two other important parameters needed to predict if a drop will be captured by a cylinder are the eccentricity of the impact, $e$, and the curvature ratio, $R^{*}$, Figure 1 . The eccentricity of impact is a measure of how off-centre the drop impact is and is defined as the distance of the drop's centre of mass from the axis of the cylinder. $R^{*}$ is the ratio of the cylinder diameter to the drop diameter. Eccentric impacts have a higher probability of occurring in actual systems, and they decrease $U_{c}[3,5,6]$. However, when the drop is impacting at velocities above $U_{c}$, eccentric impacts can increase the amount of liquid that remains on the cylinder after the drop splits $[5,6]$. Increasing $R^{*}$ increases the $U_{c}$ of droplet capture $[1,2,7,8]$. 
Within the literature, most of the studies are performed with $R^{*}<1,\left(\varnothing_{\text {drop }}>\varnothing_{\text {cylinder }}\right)$. There are only a couple of studies performed at $R^{*}>1\left(\varnothing_{\text {drop }}<\varnothing_{\text {cylinder }}\right)$ and that report eccentric impacts $[1,8]$. Within this data set, there is insufficient data within the impact velocity range of $1<\mathrm{We}<14$ and curvature ratio range $2<R^{*}<10$. In these studies, the Weber number is defined as:

$$
W e=\frac{\rho U_{0}^{2} D_{0}}{\sigma}
$$

This is equivalent to drops on the order of $10^{-5} \mathrm{~m}$ impacting cylinders with diameters on the order of $10^{-4} \mathrm{~m}$-this is seen when a fine water spray impacts a wire mesh, something we are currently studying. This experimental data is needed to validate our simulations that predict the volume of water captured by the individual wires of the wire mesh.

Our fine water spray produced droplets with an average diameter of $D_{0}=27 \pm 13 \mu \mathrm{m}$ traveling at velocities $2.8 \pm 1.6 \mathrm{~m} / \mathrm{s}$ (average $\mathrm{We}=3$ ). Since the Weber number of the sprayed drops is low, the eccentricity of impact will need to be large for the cylinder to fail in capturing the drop. We tested three general impact scenarios based on the percentage the drop overlaps the cylinder in plan view, Figure 2. For each impact scenario the impact velocity was increased from a Weber number of 1 up to the $U_{c}$. Wire diameter sizes of the mesh were $\mathrm{b}=200 \mu \mathrm{m}$ (an average $R^{*}=7.4$ ). This experiment used drop and cylinder diameters in the $\mathrm{mm}$ size range to achieve curvature ratios in the range $2<R^{*}>9$. The results are presented in regime maps for each eccentricity.

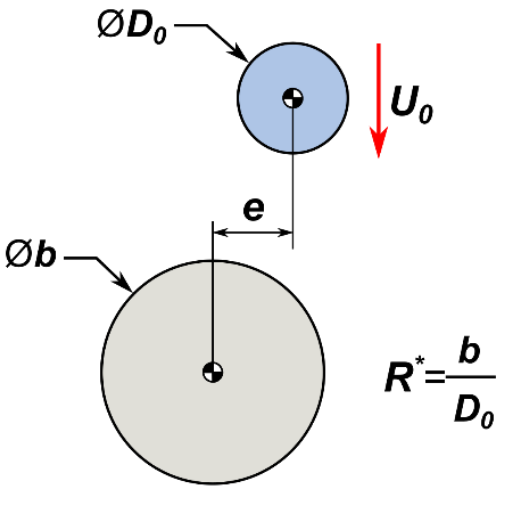

Figure 1. Schematic of droplet impact

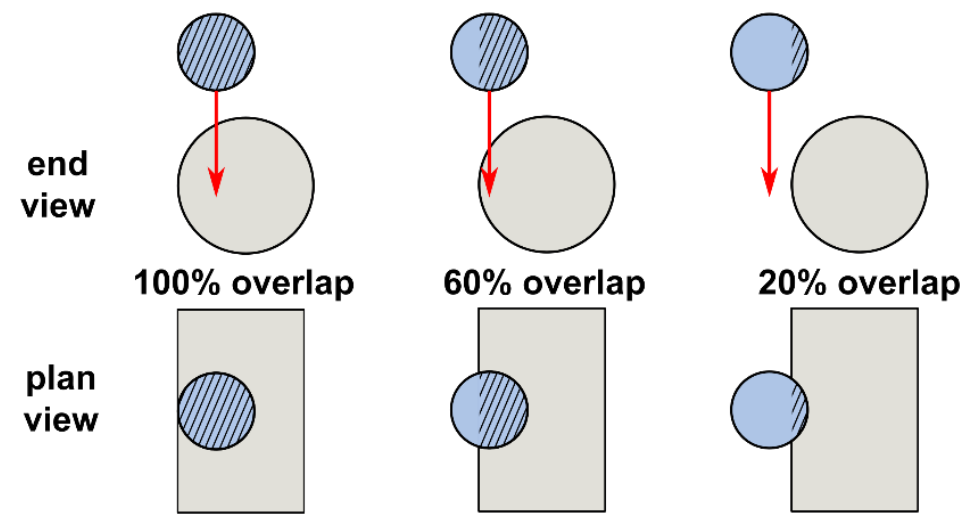

Figure 2. The percentage of droplet overlap with the cylinder for the three general impact scenarios tested.

\section{Methodology}

The cylinders that were used to impact the drops on were standard, seamless stainless steel tubes. The three sizes were nominal $25 \mathrm{~mm}, 13 \mathrm{~mm}$, and $6 \mathrm{~mm}$ stainless steel tubes each cut to $40 \mathrm{~mm}$ lengths. Each cylinder was placed in a lathe and polished with progressively finer grits of sandpaper to create a uniform surface along $75 \%$ of the cylinder length. The highest grit used was 1200. The final diameter of each cylinder was measured with calipers and reported in Table 1.

Water drops, $3.0 \mathrm{~mm}$ and $2.0 \mathrm{~mm}$, were generated from blunt-nose, $50 \mathrm{~mm}, 21$-gauge and 33-gauge syringe needles, respectively (Hamilton Company, Reno, NV, USA) connected to a syringe pump. Distilled water was used for the drops. A high-speed camera (FASTCAM 
SA5, Photron, San Diego, CA, USA) equipped with a $105 \mathrm{~mm} \mathrm{f} / 2.8$ Nikon lens and an extension bellow was positioned to capture the drop impact on the cylinders. Camera settings were $2000 \mathrm{fps}$, shutter speed ranging between 1/2000s to $1 / 4000 \mathrm{~s}$, and aperture settings between of $f / 11$ and $f / 32$. A 55 W LED light (AOS Technologies AG, 5405 Baden Daettwi, $\mathrm{CH}$ ) with a diffusing glass window was positioned directly behind the cylinder to provide backlighting.

The syringe was mounted on translation stages to provide 2-axis positioning: perpendicular to the axis of the cylinder to adjust the eccentricity of impact, and above the cylinder to vary the impact velocity. Two cylinder orientations were tested. In the "end-view" orientation, the camera was aligned to the cylinder axis. In the "side-view" orientation, the cylinder axis was turned $90^{\circ}$ to the camera lens. The drop always impacted at a fixed distance along the cylinder, approximately $25 \mathrm{~cm}$ from the nonmachined end of the cylinder. In the end-view orientation, the nonmachined end of the cylinder was positioned closest to the camera lens.

All the tests with the cylinders in the end-view orientation were tested first. Before testing each cylinder, it was triple-washed using a non-abrasive hand soap to remove oil and residue from machining, triple-rinsed with distilled water, and then dried with an air-bulb blower. The cylinder was only handled on the nonmachined end to avoid contaminating the surface. After each droplet impact was photographed, any liquid remaining on the cylinder was blotted away using lint-free lens wipes and then blown with the air-bulb blower. All the eccentricity and impact velocity combinations were tested before changing the cylinder to the next size. Drops were impacted at Weber numbers of 1, 7, and 14 three times for each impact scenario. After this base set of measurements, the height of the needle was adjusted to find the $U_{c}$ that would cause the drop to transition between completely being captured by the cylinder to partially being captured. After completing the end-view orientation measurements, the cylinder was mounted in the side-view orientation and all the measurements were repeated. This was done to obtain the spread extent of the drop in two directions. Table 1 shows the total number of tests completed, the sum of both orientations, for each impact scenario and the range of Weber numbers tested.

Table 1. Experiment test parameter ranges

\begin{tabular}{|c|c|c|c|c|}
\hline Cylinder & $\begin{array}{l}\text { Drop } \\
\text { Diameter } \\
\text { [mm] }\end{array}$ & $\begin{array}{c}\text { Curvature } \\
\text { ratio } \\
{\left[b / D_{0}\right]}\end{array}$ & $\begin{array}{c}\text { Impact scenario (e): } \\
\text { \#of tests }\end{array}$ & $\begin{array}{l}\text { Weber } \\
\text { number }\end{array}$ \\
\hline $\begin{array}{c}\text { C1 } \\
(\varnothing 6.3 \mathrm{~mm})\end{array}$ & $3.0 \mathrm{~mm}$ & 2.1 & $\begin{array}{c}100 \%(1.7 \mathrm{~mm}): \mathrm{N}=64 \\
60 \%(3.24 \mathrm{~mm}): \mathrm{N}=63 \\
20 \%(4.1 \mathrm{~mm}): \mathrm{N}=63\end{array}$ & $\begin{array}{l}1-58 \\
1-14 \\
1-14\end{array}$ \\
\hline $\begin{array}{c}\text { C2 } \\
(\varnothing 12.7 \mathrm{~mm})\end{array}$ & $3.0 \mathrm{~mm}$ & 4.2 & $\begin{array}{l}100 \%(5.0 \mathrm{~mm}): \mathrm{N}=66 \\
60 \%(6.5 \mathrm{~mm}): \mathrm{N}=60 \\
20 \%(7.3 \mathrm{~mm}): \mathrm{N}=61\end{array}$ & $\begin{array}{l}1-41 \\
1-14 \\
1-14\end{array}$ \\
\hline $\begin{array}{c}\text { C2-D } \\
(\varnothing 12.7 \mathrm{~mm})\end{array}$ & $2.0 \mathrm{~mm}$ & 6.3 & $\begin{array}{c}100 \%(5.5 \mathrm{~mm}): \mathrm{N}=61 \\
60 \%(6.0 \mathrm{~mm}): \mathrm{N}=64 \\
20 \%(6.8 \mathrm{~mm}): \mathrm{N}=60\end{array}$ & $\begin{array}{l}1-44 \\
1-26 \\
1-16\end{array}$ \\
\hline $\begin{array}{c}\text { C3 } \\
(\varnothing 25.4 \mathrm{~mm})\end{array}$ & $3.0 \mathrm{~mm}$ & 8.4 & $\begin{array}{l}100 \%(11.0 \mathrm{~mm}): \mathrm{N}=63 \\
60 \%(12.5 \mathrm{~mm}): \mathrm{N}=60 \\
20 \%(13.3 \mathrm{~mm}): \mathrm{N}=60\end{array}$ & $\begin{array}{l}1-43 \\
1-28 \\
1-20\end{array}$ \\
\hline
\end{tabular}

Images of the impacting drops were processed in MATLAB using a custom automated script that first converted each image into a binary black and white image. The droplet was measured to calculate the initial volume and track the change in position of the drop's centre of mass 5 frames $(2.5 \mathrm{~ms})$ prior to impact to calculate the impact velocity. The scale used to 
process the images is estimated to be accurate within 2 pixels and the location of the drop's boundary is estimated to be accurate within 2 pixels. The uncertainty in the drop diameters is $2.0 \pm 0.08 \mathrm{~mm}$ and $3.0 \pm 0.11 \mathrm{~mm}$. The uncertainty in the drop's initial volume is $4.2 \pm 0.53 \mu \mathrm{l}$ and $14.1 \pm 1.6 \mu \mathrm{l}$ for the $2.0 \mathrm{~mm}$ and $3.0 \mathrm{~mm}$ drops, respectively. All the photos presented in this paper were cropped and adjusted for contrast and brightness in ImageJ [9].

\section{Observations}

The effect of the curvature ratio on the $U_{c}$ of a drop is shown in Figure 3. The cylinder diameter, the Weber number, and dimensionless eccentricity $\left.\left(e^{*}=e /\left\{\left[D_{0}+b\right) / 2\right]\right\}\right)$ of the impacting drop are held constant at $12.5 \mathrm{~mm}, \mathrm{We}=14$, and $e^{*}=0.82$, respectively. In Figure $3 a$, only the portion of the drop that is overlapping with the cylinder adheres to the cylinder while the portion of the drop that projects beyond the cylinder's edge continues to travel both down past the cylinder, $8 \mathrm{~ms}$. The inertia within this portion of the drop causes it to elongate as the drop travels past the cylinders midplane, but surface tension forces begin to resist this movement and begins to draw the drop back towards the cylinder, $16 \mathrm{~ms}$ and $24 \mathrm{~ms}$. At 32 $\mathrm{ms}$, the inertia in the free portion of the drop is still causing it to travel down and towards the cylinder's central axis, and the drop begins to neck again because the mass of the drop exceeds the resisting surface tension forces. By $40 \mathrm{~ms}$, the drop splits into two.

In Figure 3b, the $2.0 \mathrm{~mm}$ drop begins impacting the cylinder similar to the $3.0 \mathrm{~mm}$ drop in Figure 3a, but it has less mass projecting beyond the cylinder's edge and ultimately less inertia to elongate the drop as it travels past the midplane of the cylinder, $8 \mathrm{~ms}$. As the drop continues to travel around the perimeter of the cylinder, surface tension forces are greater than the drop's momentum and the entire drop is brought in and adheres to the cylinder, 16$40 \mathrm{~ms}$. The curvature ratio can also be increased by increasing the diameter of the cylinder. In this scenario, the drop would impact higher up on the cylinder and have to travel a greater arclength distance before reaching the midplane of the cylinder. This extra travel distance dissipates more energy and provides more time for the drop to rotate into the cylinder because immediately upon impact surface tension forces tend to pull the drop towards the central axis of the cylinder. This decreases the mass of liquid that projects beyond the cylinder and shrinks the force that surface tension has to resist as the drop travels past the midplane of the cylinder. 
(a)

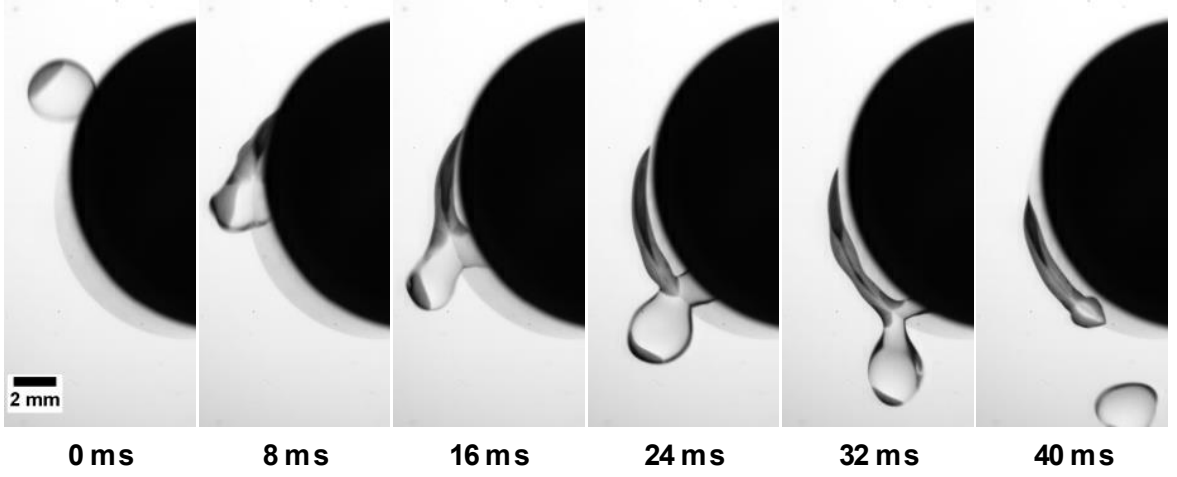

(b)

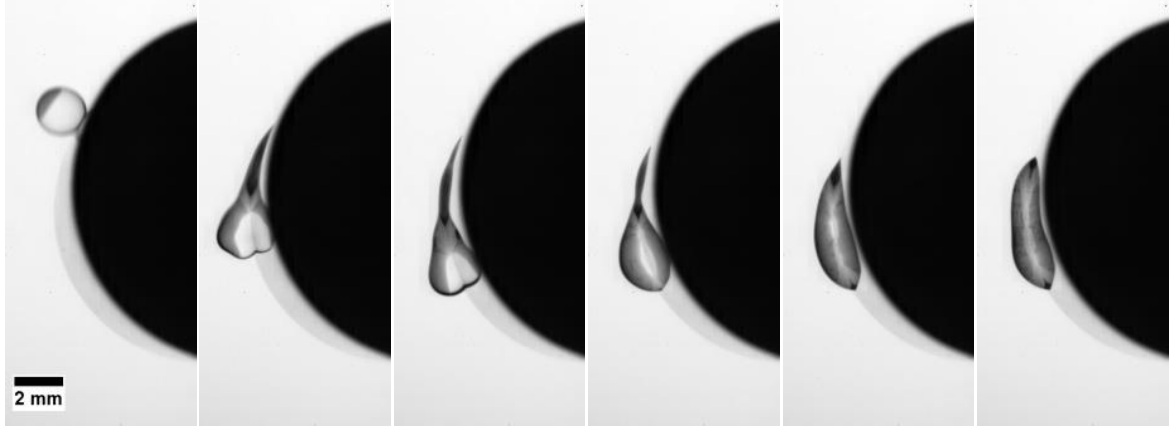

Figure 3. Increasing the curvature ratio increases the $U_{c}$ of capture for a droplet on a cylinder. The diameter of the cylinder is $12.5 \mathrm{~mm}$, the Weber number, $\mathrm{We}=14$, and the dimensionless eccentricity of impact, $e^{*}=0.82$, for the two scenarios shown. The $3.0 \mathrm{~mm}$ drop impacting in (a) has a curvature ratio $R^{\star}=4.2$. The $2.0 \mathrm{~mm}$ drop impacting in (b) has a curvature ratio of $R^{*}=6.3$

Figure 4 shows the effect of increasing the eccentricity of impact on the $U_{c}$ of a drop. The curvature ratio and the Weber number are held constant at $R^{*}=4.2$ and $\mathrm{We}=7$, respectively, for the three scenarios shown. The eccentricity of impact shown in Figure $4 a$ is representative of the 100\% drop overlap scenario. The drop strikes high on the cylinder and must travel the greatest arclength to reach the cylinder's midplane. Also, in this scenario, only a small volume of liquid elongates past the edge of the cylinder, $12 \mathrm{~ms}$. It is quickly resisted and pulled back towards the cylinder by surface tension forces by $18 \mathrm{~ms}$. At high impact velocities, the curvature of the cylinder can cause a mass of liquid to accelerate away from the cylinder central axis with enough momentum that the drop shown in the $12 \mathrm{~ms}$ frame of Figure $4 \mathrm{a}$ will continue to elongate and split.

The eccentricity of impact shown in Figure $4 \mathrm{~b}$ represents the $60 \%$ drop overlap scenario. It strikes the cylinder closer to the cylinder's midplane and has a smaller arclength distance to travel than the drop in Figure 4a. The combination of some of the drop projecting beyond the cylinder's edge and a shorter arclength to travel results in more mass to elongate the drop, compare Figure $4 \mathrm{~b}$ with Figure $4 \mathrm{a}$ at $12 \mathrm{~ms}$. However, the inertia in this mass is still smaller than the resisting surface tension forces and the drop is fully captured by the cylinder, with a final resting position below the midplane of the cylinder.

Figure 4c shows the greatest eccentricity of impact and represents the $20 \%$ drop overlap scenario. Here, the drop impacts closest to the cylinder midplane and has the greatest portion of the drop projecting beyond the edge of the cylinder. As the drop travels past the midplane of the cylinder, surface tension forces pull the drop towards the cylinder's central axis, wetting the underside of the cylinder and slowing the drop (18-30 ms); however, there 
is insufficient surface tension forces to fully resist the inertia of the free portion of the drop and it eventually necks and splits into two (36 ms).

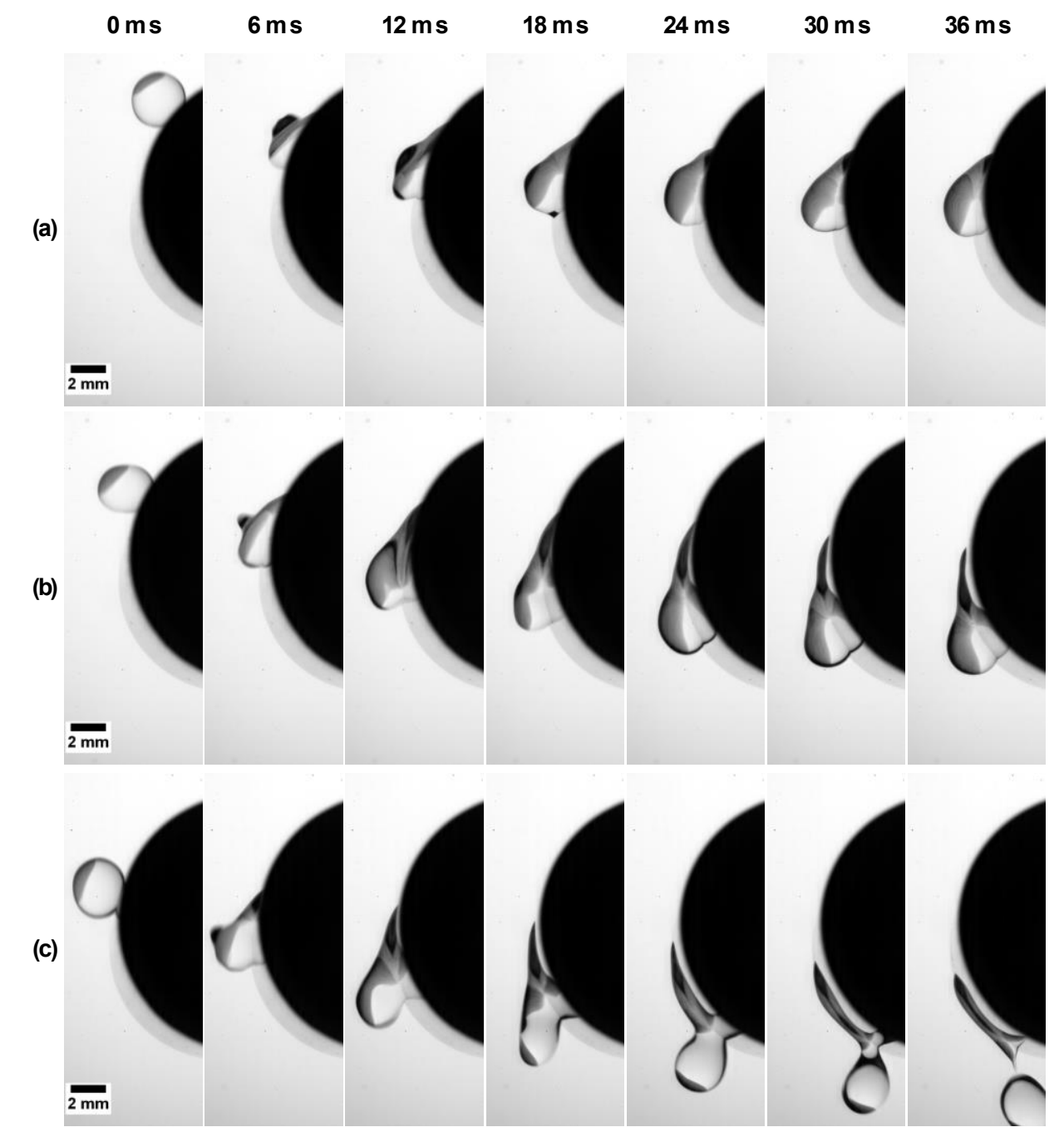

Figure 4. Increasing the eccentricity of impact decreases $U_{c}$ of capture for a drop on a cylinder. The diameter of the cylinder is $12.5 \mathrm{~mm}$, diameter of the drop is $3.0 \mathrm{~mm}\left(R^{*}=4.2\right)$, and the Weber number, We $=7$, for the three scenarios shown. The $e^{*}=0.65(e=5.0 \mathrm{~mm})$ for $(\mathrm{a}), e^{*}=0.84(e=6.5 \mathrm{~mm})$ for $(\mathrm{b})$, and $e^{*}=0.95(e=7.3 \mathrm{~mm})$ for $(\mathrm{c})$

Figure 5 plots all of the experimental data with the impact Weber number against the curvature ratio for the three general impact scenarios. The blue dashed lines in Figure 5 denote the general separation between the complete capture of the drop region from the drop splitting region. We find that the $U_{c}$ increases with an increasing curvature ratio.

Additionally, as the eccentricity of impact increases, the curvature ratio has a larger effect on the $U_{c}$, indicated by the increasing slope of the blue dashed lines as the percentage of overlap decreases from $100 \%$ to $20 \%$. Increasing the eccentricity of droplet impact decreases the $U_{c}$ of droplet capture. This is observed in Figure 5 as the downward shift of the blue dashed line as the percentage of overlap decreases from the $100 \%$ to $20 \%$ (increasing eccentricity). Our results are in general agreement with the existing literature for eccentric droplet impacts at low curvature ratios $\left(R^{*}<1\right)$. Analysis of the volume captured by the wire when the drop splits and the comparison of these experiments to analytical models is the subject of future work. 

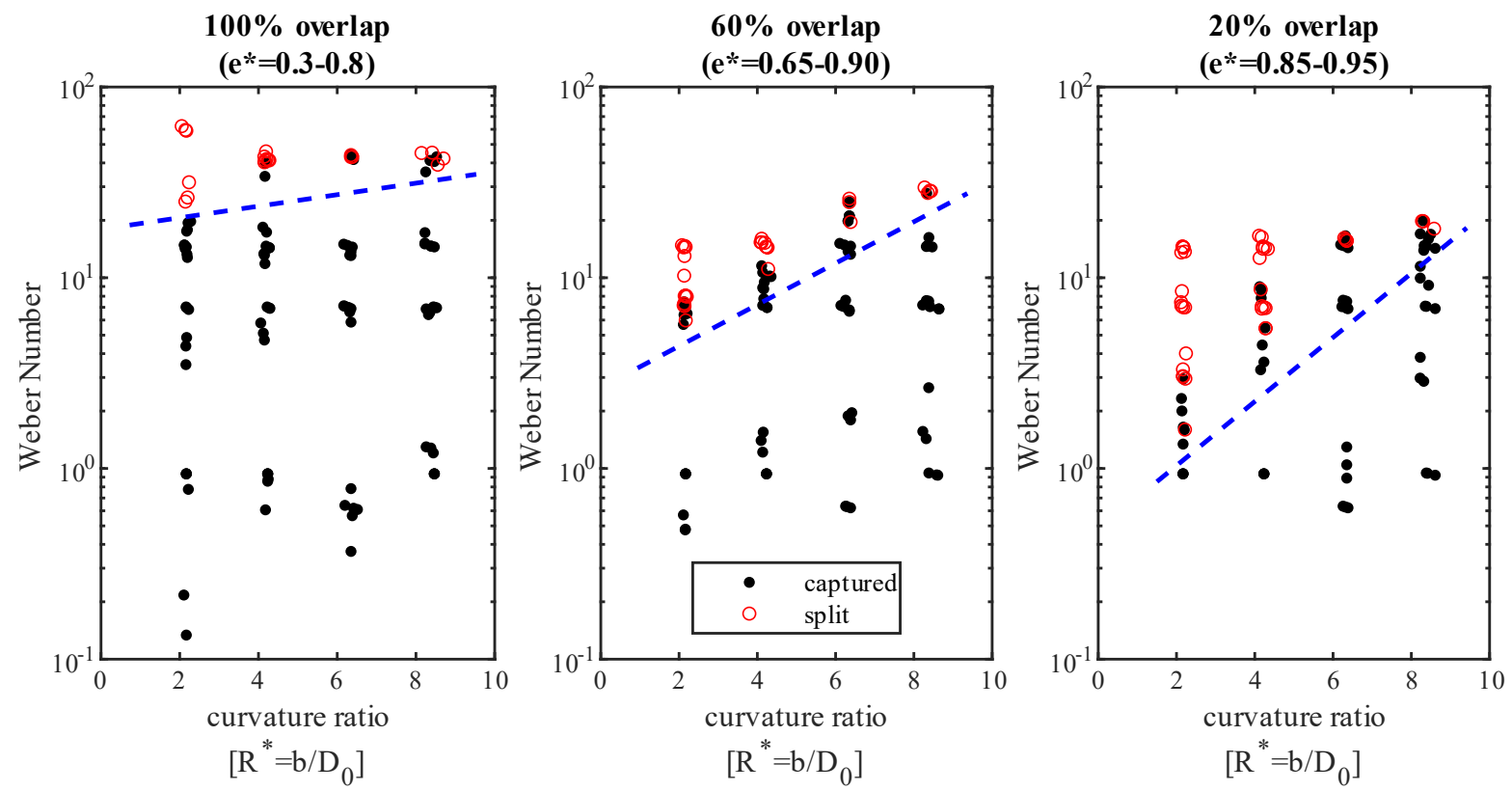

Figure 5. Regime map for impact Weber number against the curvature ratio, sorted into the three impact scenarios tested. Each impact scenario covers a range of eccentricities. Increasing the curvature ratio increased the $U_{c}$ of drop capture in all impact scenarios. In general, increasing the eccentricity of impact decreased the $U_{c}$ of drop capture

\section{Conclusions}

Our results add to the existing data in the literature by providing threshold $U_{c}$ data in the range of $1<\mathrm{We}<14$ and $2<R^{*}<9$. The threshold velocity of capture for an impacting water drop on a cylinder increases with increasing curvature and decreases with increasing eccentricity. This in agreement with the general trends for the $U_{c}$ reported in the literature for eccentric droplet impacts at $R^{*}<1$. We show for the first time the $U_{c}$ where a drop fails to be captured by a cylinder in this low impact velocity, high curvature ratio regime.

\section{Acknowledgments}

The authors would like to acknowledge the contribution of HengSheng (Ben) Yang, who gathered the experimental data for the end-view orientation cylinder impacts. 


\section{Nomenclature}

$b \quad$ wire/cylinder diameter [mm]

$D_{0} \quad$ drop diameter [mm]

$e \quad$ eccentricity of impact [mm]

$e^{*} \quad$ dimensionless eccentricity $\left[e^{*}=e /\left(\left(D_{0}+b\right) / 2\right)\right]$

$R^{*} \quad$ curvature ratio $\left[R^{*}=b / D_{0}\right]$

$U_{0} \quad$ impact velocity [ $\left.\mathrm{m} \mathrm{s}^{-1}\right]$

$U_{c} \quad$ threshold impact velocity $\left[\mathrm{m} \mathrm{s}^{-1}\right]$

We Weber number $\left[\rho U_{0}^{2} \mathrm{D}_{0} / \sigma\right]$

$\varnothing \quad$ diameter [mm]

$\rho \quad$ density $\left[\mathrm{kg} \mathrm{m}^{-3}\right]$

$\sigma \quad$ surface tension $\left[\mathrm{N} \mathrm{m}^{-1}\right]$

\section{References}

[1] Hung, L. S., and Yao, S. C., 1999, "Experimental Investigation of the Impaction of Water Droplets on Cylindrical Objects," Int. J. Multiph. Flow, 25(8), pp. 1545-1559.

[2] Lorenceau, É., Clanet, C., and Quéré, D., 2004, "Capturing Drops with a Thin Fiber," J. Colloid Interface Sci., 279(1), pp. 192-197.

[3] Safavi, M., and Nourazar, S. S., 2018, "Experimental, Analytical, and Numerical Study of Droplet Impact on a Horizontal Fiber," Int. J. Multiph. Flow, (xxxx).

[4] Kim, S. G., and Kim, W., 2016, "Drop Impact on a Fiber," Phys. Fluids, 28(4).

[5] Lorenceau, E., Clanet, C., Quéré, D., and Vignes-Adler, M., 2009, "Off-Centre Impact on a Horizontal Fibre," Eur. Phys. J. Spec. Top., 166(1), pp. 3-6.

[6] Sher, E., Haim, L. F., and Sher, I., 2013, "Off-Centered Impact of Water Droplets on a Thin Horizontal Wire," Int. J. Multiph. Flow, 54, pp. 55-60.

[7] Kim, S. G., and Kim, W., 2016, "Drop Impact on a Fiber," Phys. Fluids, 28(4).

[8] Pasandideh-Fard, M., Bussmann, M., and Chandra, S., 2001, "Simulating Droplet Impact on a Substrate of Arbitrary Shape," At. Sprays, 11(4), pp. 397-414.

[9] Schneider, C. A., Rasband, W. S., and Eliceiri, K. W., 2012, "NIH Image to ImageJ: 25 Years of Image Analysis," Nat. Methods, 9(7), pp. 671-675. 